\title{
Alchemical perturbation density functional theory
}

\author{
Guido Falk von Rudorff $\odot$ and O. Anatole von Lilienfeld $\odot^{*}$ \\ Institute of Physical Chemistry and National Center for Computational Design and Discovery of Novel Materials (MARVEL), \\ Department of Chemistry, University of Basel, Klingelbergstrasse 80, CH-4056 Basel, Switzerland
}

(Received 12 September 2018; revised manuscript received 13 March 2019; accepted 30 March 2020; published 22 May 2020)

\begin{abstract}
We introduce an orbital-free electron density functional approximation based on alchemical perturbation theory. Given convergent perturbations of a suitable reference system, the accuracy of popular self-consistent Kohn-Sham density functional estimates of properties of new molecules can be systematically surpassed-at negligible cost. The associated energy functional is an approximation to the integrated energy derivative, requiring only perturbed reference electron densities: No self-consistent field equations are necessary to estimate energies and electron densities. Electronic ground state properties considered include covalent bonding potentials, atomic forces, noncovalent interactions, and dipole and quadrupole moments.
\end{abstract}

DOI: 10.1103/PhysRevResearch.2.023220

\section{INTRODUCTION}

With the success of electronic structure methods in materials, chemical, and biological sciences, the need for ever more accurate yet ever computationally more affordable methods has grown. The Harris functional [1,2] employed an approximate density rather than a self-consistent one by following the Kohn-Sham scheme [3] for one step only. While yielding energies with acceptable accuracy for bulk crystals [4,5], density differences are significant [6] and resulting energies are neither upper nor lower bounds to the self-consistent energy $[7,8]$. This has been attributed to the nonvariational approach and addressed by treating the approximate wave function as perturbation to the true wave function $[9,10]$. For applications however, this concept has faced technical difficulties [11] and depends strongly [11] on the quality of the approximate density which often has been obtained by superimposing fragment densities. Nevertheless, this approach has been useful in improving convergence [11]. Other approaches have been introduced by Foldy, Wilson, Reif, Frost, and Daza [12-16]. The transition functional method of Nagy [17] gives energy differences of two molecules if both their electron densities are known. Similar approaches have helped address the problem of a reliable kinetic energy expression in the context of orbital-free density functional theory (DFT) [18-21].

If densities change smoothly along the isoelectronic integration path, the mean value theorem mandates that evaluating the integrand once for one (unknown) point on the integration path is sufficient to obtain an accurate energy [22]. Based on scaling nuclear charges, a relation for the ground state energy

\footnotetext{
*anatole.vonlilienfeld@unibas.ch

Published by the American Physical Society under the terms of the Creative Commons Attribution 4.0 International license. Further distribution of this work must maintain attribution to the author(s) and the published article's title, journal citation, and DOI.
}

as a function of the electrostatic potential at the nuclei was given [23]. It has been suggested to expand the total energy in polynomials of the nuclear charges [24], which converges quickly for small systems [25] and treats the nuclei-electrons and electron-electron interactions [26]. The model was used to show that the electron-electron interaction energy is limited in isoelectronic molecular series [27], and to propose bounds on neutral atom energies [28].

More recently, alchemical perturbations in the spirit of Foldy [12] and Wilson [13] have gained traction. In analogy to adiabatic coupling and the integration paths between molecules in the Wilson scheme [22,29], the electronic Hamiltonians of two (isoelectronic) systems are adiabatically coupled via an arbitrary path described by a mixing parameter. The change in nuclear charges then can be considered as perturbation of one endpoint [30,31]. Although this perturbation is by no means small, the approach was successful in screening of alkali halide crystals [32]; estimating the chemical potential of binary mixtures [33]; calculating bond potentials [34-36]; estimating energies, structures and volume in solid metals [37]; band structures in III-V semiconductors [38]; predicting reaction barriers and molecular adsorption on metals $[39,40]$; mixed-metallic clusters [41]; predicting adsorption energy changes of water on graphene due to $\mathrm{BN}$ doping [42]; calculating higher-order energy derivatives [43]; exploring chemical space $[44,45]$; predicting $\mathrm{BN}$-doped $\mathrm{C}_{60}$ [46]; and probing the nonlocal nature of the electron density [47]. By contrast, we describe the application of perturbation theory to estimate energies and electron density, resulting in a consistent orbital-free alchemical perturbation density functional theory of order $n$ (APDFT $n$ ). Given a single reference electron density and energy, accurate electron densities and energies of isoelectronic systems with identical nuclear positions are obtained at negligible computational cost.

This is achieved via alchemical perturbation, i.e., coupling two electronic molecular Hamiltonians $\hat{H}_{\mathrm{t}}$ and $\hat{H}_{\mathrm{r}}$ via the linear mixing parameter $\lambda$ as $\hat{H}(\lambda) \equiv \lambda \hat{H}_{\mathrm{t}}+(1-\lambda) \hat{H}_{\mathrm{r}}$. The resulting energy $E_{\mathrm{t}}$ for a target system can be expanded in 
a Taylor series around the reference molecule (i.e., $\lambda=0$ ) $E_{\mathrm{t}} \equiv E(\lambda=1)$, which can be expressed as

$$
\begin{aligned}
E_{\mathrm{t}} & =\sum_{n=0}^{\infty} \partial_{\lambda}^{n}\left\langle\psi_{\lambda}|\hat{H}(0)| \psi_{\lambda}\right\rangle /\left.n !\right|_{\lambda=0} \\
& =\left.\sum_{n=0}^{\infty} \frac{1}{n !} \frac{\partial^{n} E(0)}{\partial \lambda^{n}}\right|_{\lambda=0}=E_{\mathrm{r}}+\left.\sum_{n=1}^{\infty} \frac{1}{n !} \frac{\partial^{n} E(0)}{\partial \lambda^{n}}\right|_{\lambda=0} .
\end{aligned}
$$

With the Hellmann-Feynman theorem [48], the first-order derivative can be expressed as [31]

$$
\frac{\partial E(\lambda)}{\partial \lambda}=\left\langle\psi_{\lambda}\left|\hat{H}_{\mathrm{t}}-\hat{H}_{\mathrm{r}}\right| \psi_{\lambda}\right\rangle=\int d \mathbf{r} \underbrace{\left[v_{\mathrm{t}}(\mathbf{r})-v_{\mathrm{r}}(\mathbf{r})\right]}_{\equiv \Delta v} \rho_{\lambda}(\mathbf{r}),
$$

where $\psi_{\lambda}$ is the self-consistent wave function for a given value of $\lambda$ with density $\rho_{\lambda}$. Higher-order derivatives come from further differentiation: $\partial_{\lambda}^{n} E(\lambda)=\int d \mathbf{r} \Delta v \partial_{\lambda}^{(n-1)} \rho_{\lambda}$. Insertion into Eq. (1) gives the following for $E_{\mathrm{t}}-E_{\mathrm{r}}$,

$$
\left.\sum_{n=1}^{\infty} \frac{1}{n !} \int d \mathbf{r} \Delta v \frac{\partial^{n-1} \rho_{\lambda}}{\partial \lambda^{n-1}}\right|_{\lambda=0}=\int_{\Omega} d \mathbf{r} \Delta v \underbrace{\left.\sum_{n=1}^{\infty} \frac{1}{n !} \frac{\partial^{n-1} \rho_{\lambda}}{\partial \lambda^{n-1}}\right|_{\lambda=0}}_{\equiv \tilde{\rho}},
$$

where we switch sum and integral assuming uniform convergence of the sum since the integral can be restricted to finite volume $\Omega$, either because both densities (target and reference) are bound or because periodicity requires a finite unit cell.

This gives a new electron density $\tilde{\rho}$, which we can understand using thermodynamic integration for $E_{\mathrm{t}}-E_{\mathrm{r}}$ :

$$
\begin{aligned}
\int_{0}^{1} d \lambda \frac{\partial E}{\partial \lambda} & =\int_{\Omega} d \mathbf{r} \Delta v(\mathbf{r}) \int_{0}^{1} d \lambda \rho_{\lambda}(\mathbf{r}) \\
& =\int_{\Omega} d \mathbf{r} \Delta v(\mathbf{r}) \tilde{\rho}(\mathbf{r}) .
\end{aligned}
$$

Expansion of $\rho_{\lambda}$ as a Taylor series, $\rho_{\mathrm{t}} \equiv \rho(\lambda=1)=\rho_{\mathrm{r}}+$ $\sum_{n=1}^{\infty} \frac{1}{n !} \partial_{\lambda}^{n} \rho(0)$, recovers Eq. (3). The $\lambda$-averaged density $\tilde{\rho}$ is neither the density of the reference nor the target. While Levy approximated $\tilde{\rho}$ by averaging $\rho_{r}$ and $\rho_{t}$ [22], we focus on its Taylor expansion in $\rho_{r}$, which can crucially account for nonlinearities while rendering the computational investment constant and independent of target system.

For $\mathrm{N}_{2}$, we exemplify $\tilde{\rho}$ in Fig. 3. Note that Eq. (4) implies convergence in $\lambda$ as long as $\partial_{\lambda} E$ does not diverge. While we show convergence for hydrogenic atoms and free atoms (see Supplemental Material [49]), we cannot offer a rigorous proof of convergence for all systems. It is likely, however, that the electron density can be described by an analytic function, i.e., a function with a converging Taylor series, since the electron density is commonly approximated with Gaussian functions in quantum chemistry, both for orbitals and machine learning [50]. For the many cases where this approximation is valid, any density derivative is analytic, which means that $\tilde{\rho}$ converges. As shown numerically below, this sum can be truncated early. This gives an energy functional that only depends on the reference electron density $\rho_{\mathrm{r}}(\mathbf{r})$ and its perturbations in nuclear charge, or pseudopotential parameters [35,38], which

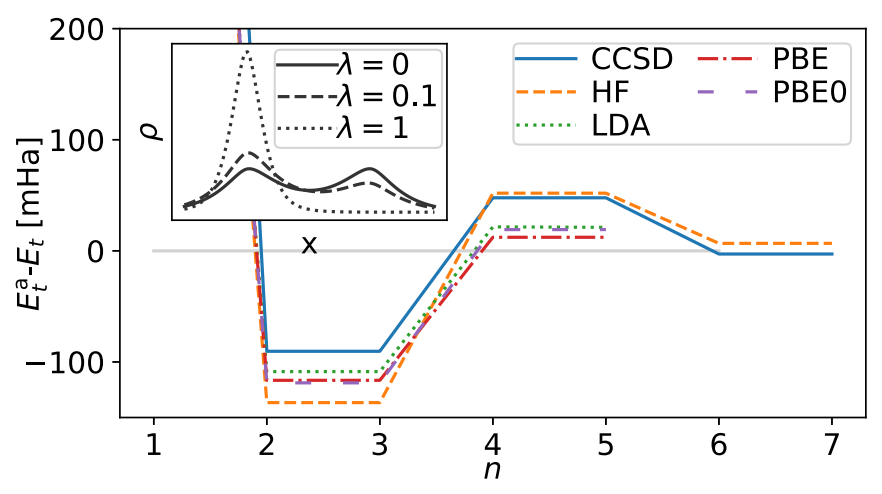

FIG. 1. Deviation of APDFTn energy $\left(E_{t}^{a}\right)$ from self-consistent field energy $\left(E_{t}\right)$ according to Eq. (3) for various levels of theory (see Supplemental Material [49] for details). Reference and target systems are $\mathrm{H}_{2}(d=1 \AA)$ and $\mathrm{He}$, respectively. Inset: Electron density along the molecular axis.

can be connected to a change in $\lambda$ through the chain-rule:

$$
\frac{\partial^{2} \rho}{\partial \lambda^{2}}=\frac{\partial \rho}{\partial \lambda} \sum_{I} \frac{\partial \rho}{\partial Z_{I}} \frac{\partial Z_{I}}{\partial \lambda}=\sum_{J} \frac{\partial^{2} \rho}{\partial Z_{I} \partial Z_{J}} \frac{\partial Z_{I}}{\partial \lambda} \frac{\partial Z_{J}}{\partial \lambda},
$$

with $Z_{I}$ as the nuclear charge of atom $I$. This approach could be extended to nonisoelectronic systems through fractional numbers of electrons. However, due to the derivative discontinuities with respect to the electron number, we expect much worse performance.

We find it exciting that no quantum calculation is necessary for the target molecule. Its specific chemistry enters solely via analytically known terms, the nuclear repulsion energy and $\Delta v(\mathbf{r})$. While interesting in general, such a functional is particularly useful for large screening calculations where many similar molecules have to be assessed very quickly, since APDFT requires one self-consistent density only.

\section{RESULTS}

To test how fast the above equations converge, we first estimated the energy of $\mathrm{He}$ using perturbations for $\mathrm{H}_{2}$. Figure 1 shows the resulting energy estimate errors as a function of the highest order included in the Taylor expansion for this twoelectron system. Regardless of the reference method [HartreeFock (HF), local density approximation (LDA), generalized gradient approximation (GGA), Hybrid-GGA, coupled cluster single double (CCSD)], the error of the perturbative approach is reduced systematically with higher-order terms. We observe numerical noise for higher orders only: from order 5 for methods using a DFT grid and from order 7 for those without. Due to spatial symmetry, even orders in the expansion do not contribute due to integrand parity. This example also highlights that vanishing nuclei can be treated without any adjustment to the method [31].

Going from toy model systems to more relevant molecules, we have estimated the binding energy of $\mathrm{CO}$ perturbing the electron density of $\mathrm{N}_{2}$ using APDFT2. Figure 2 shows the results over a wide range of interatomic distances for various levels of theory used for the reference calculation. APDFT2 consistently gives numbers close to the actual potential energy curve for any given level of theory that has been used to 

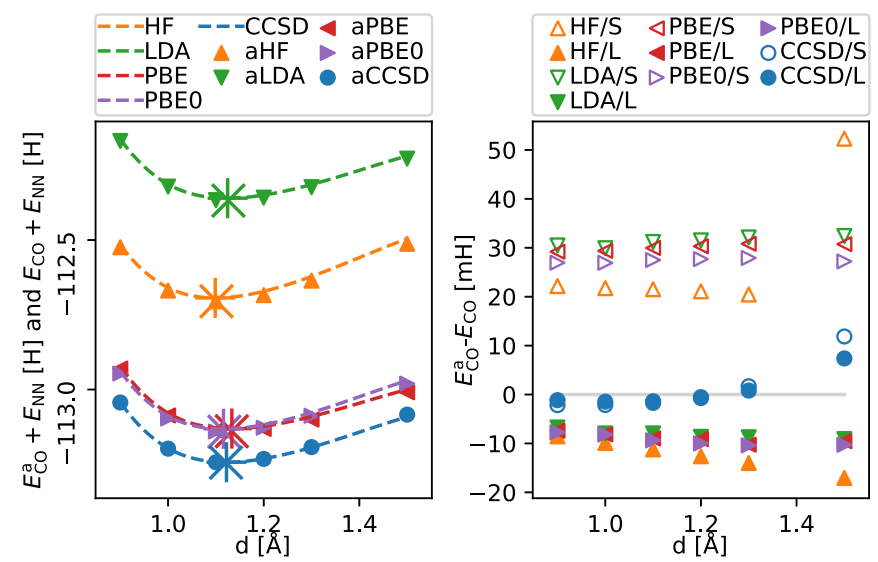

FIG. 2. True ( $E_{\mathrm{CO}}$, dashed) and APDFT2 based ( $E_{\mathrm{CO}}^{\mathrm{a}}$, symbols) binding potentials of $\mathrm{CO}$ for various methods using $\mathrm{N}_{2}$ as a reference (details in Supplemental Material [49]). Left: Total potentials (including nuclear repulsion $E_{\mathrm{NN}}$ ). Plus/cross symbols: Equilibrium distances for target/alchemy, respectively. Right: Respective deviation for small [S =6-31G(d)] and large [L= def2-TZVP] basis sets obtained from $\mathrm{N}_{2}$ and reference potential at the same level of theory $E_{\mathrm{CO}}$ (dashed lines).

derive the electron density around the reference molecule. This highlights that APDFT2 approximates the energy of the reference level of theory (rather than the true energy). Moreover, the location of the minima of the covalent bonding curves of CO in Fig. 2 are nearly identical for both APDFT2 and the reference calculations.

Following the potential energy surface over the course of a bonding curve covers a significant potential energy range and requires systematic accuracy for intermediate distances. Figure 2 shows the difference between APDFT2 predictions and the energy of the self-consistent density. Over a wide range of bond distances, the approximate potential energies are accurate to $20-30 \mathrm{mH}$ for a small basis set, while a larger basis set yields an accuracy of about $-10 \mathrm{mH}$. This is different for CCSD densities where-regardless of basis setthe accuracy is some $2 \mathrm{mH}$ at second order for nondissociative geometries, as shown in Fig. 2. The systematic error exhibited for all levels under investigation shows the consistency of APDFT2. This shows that the energy estimates as obtained from APDFT2 are significantly better than the ones obtained from established methods (see application examples below or Supplemental Material [49] for further details), which suggests employing few alchemical perturbations at a higher level of theory rather than many lower-level calculations. This is with the exception of HF for a small basis set and a bond distance of $1.5 \AA$, where the numerical differentiation introduces artifacts. Note that the finite difference scheme for density derivatives is no requirement but rather has been used for proof-of-concept work.

In all test cases, larger basis sets yield more accurate potential energies. This is partially because the overlap of the basis set decreases with increasing bond length, which offers fewer degrees of freedom for electron density to follow the change in nuclear charges. The major contribution comes from the finite number of expansion terms. While we have no proof, the expansion appears to converge faster for a larger

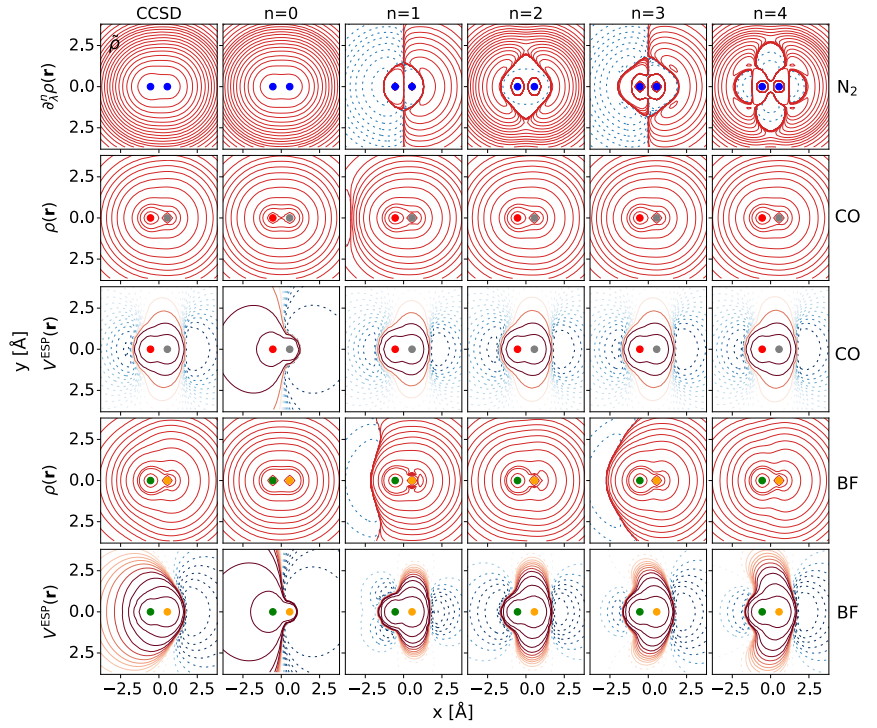

FIG. 3. Top row: Left-hand panel shows $\tilde{\rho}$ for alchemically transforming $\mathrm{N}_{2}$ into CO [Eq. (3)], estimated by $n$ th-order electron density derivatives (other panels). Contour levels (red solid lines are positive; blue dashed lines are negative) are shown for CCSD (leftmost column) and APDFTn-based estimates of $\mathrm{CO}$ and BF electron densities $(\rho)$ and electrostatic potentials $(V)$ (details are given in the Supplemental Material [49]). Convergence is shown in Table I.

basis set. Second-order perturbation of $\mathrm{N}_{2}$ yields significantly worse results for BF. This is not surprising since the electron density changes are more dramatic. Inclusion of third-order terms results in improved binding potentials (see Supplemental Material [49]). For comparison, we have also calculated covalent bonding energies in $\mathrm{CO}$ and BF using Levy's density averaging approach [22], which deviate by up to $\approx 0.6$ and 2.5 hartree from the CCSD numbers (see Supplemental Material [49]).

Overall, our results suggest that investing in high-level and higher-order perturbations of suitable reference systems enables high-throughput-screening of alchemically related materials-with acceptable accuracy. The number of accessible targets scales faster than the cost of higher-level methods. For example, for $(\mathrm{BN})_{n}$-doped graphene with $N$ atoms the total number of targets grows binomially $\left(\sum_{n=1}^{N}\left(\begin{array}{c}N \\ 2 n\end{array}\right)\left(\begin{array}{c}2 n \\ n\end{array}\right) \simeq\right.$ $\frac{3^{N}}{5}$ ), rather than polynomially.

Figure 3 illustrates the predictive power of APDFT $n$ for various electronic properties of $\mathrm{CO}$ and $\mathrm{BF}$ (calculated from $\mathrm{N}_{2}$ ). Unsurprisingly, estimates for $\mathrm{CO}$ converge more quickly than for BF. This is expected since nuclear charge changes are greater for $\mathrm{BF}$ than for $\mathrm{CO}$ (larger domain to be covered by Taylor expansion). The limited accuracy of multipole moments (see Table I) can be understood when considering the long-range electrostatic potential in Fig. 3: Outer regions converge slower with expansion order. Consistently, APDFTnbased predictions of local properties, such as ionic forces, converge faster. As shown in Table I (and in the Supplemental Material [49]), HF and the different DFT functionals perform similarly for all cases. In comparison to CCSD, however, alchemical predictions are often more accurate than their DFT 
TABLE I. Electronic dipole moments $\mu$ and quadrupole moments $Q_{\mathrm{xx}}$, and ionic forces $F$ of $\mathrm{CO}(d=1.1 \AA$, see Supplemental Material [49] for $1.5 \AA$ and $\mathrm{BF}$ results) obtained from reference CCSD, APDFTn (see Fig. 3), HF, LDA, PBE, and PBE0 densities. Errors $\delta$ are given relative to CCSD.

\begin{tabular}{lcccccr}
\hline \hline Method & $|\boldsymbol{\mu}|$ & $\delta|\boldsymbol{\mu}|(\%)$ & $Q_{\mathrm{xx}}$ & $\delta Q_{\mathrm{xx}}(\%)$ & $|\mathbf{F}|$ & $\delta|\mathbf{F}|(\%)$ \\
\hline CCSD & 12.54 & & -27.57 & & 10.96 & \\
APDFT0 & 14.55 & 16.05 & -31.37 & 13.76 & 12.96 & 18.22 \\
APDFT1 & 12.48 & -0.45 & -27.09 & -1.77 & 11.07 & 0.99 \\
APDFT2 & 12.49 & -0.41 & -27.46 & -0.40 & 10.95 & -0.09 \\
APDFT3 & 12.52 & -0.12 & -27.55 & -0.08 & 10.96 & -0.04 \\
HF & 12.42 & -0.92 & -27.43 & -0.50 & 10.82 & -1.33 \\
LDA & 12.60 & 0.47 & -27.67 & 0.37 & 10.91 & -0.53 \\
PBE & 12.60 & 0.49 & -27.70 & 0.46 & 10.85 & -1.02 \\
PBE0 & 12.54 & 0.01 & -27.59 & 0.05 & 10.85 & -1.03 \\
\hline \hline
\end{tabular}

counterparts. Table I shows all numerically stable alchemical predictions up to order 3. Due to the nature of the density expansion, negative electron densities can arise for intermediate values. While negative electron densities are unphysical, the Taylor expansion does not restrict the sign. Negative densities, however, indicate a lack of convergence, as illustrated by the improvement from order 1 to 3 in the BF case.

It should be obvious that APDFT is not a black-box method: While we have used closely related reference and target systems in the above examples, the careful choice of reference system is crucial, as in any perturbation-theory-based method. More fundamental limitations arise from the requirement that the density response be continuous. The $\mathrm{H}_{2}{ }^{+}$system represents an exemplary pathological breakdown where a small perturbation of the molecular symmetry results in abrupt changes in the entire electron density [35,51]. Or, scaling down nuclear charges can lead to discontinuous density responses of unbound electrons [52]. A technical requirement for $\partial_{\lambda} \rho$ to be smooth is that the atomic basis functions overlap sufficiently, as illustrated by the distance dependency in Fig. 2. Details on the central finite difference scheme and numerical integration are given in the Supplemental Material [49].

Recently, an exhaustive enumeration of all the 7.4 tera BNdoped two-dimensional graphitic molecules up to coronene [55] was reported with DFT estimates only for a tiny subset due to the associated cost. We have investigated the applicability of APDFT for solving such computational challenges in two related applications: First, we performed an exhaustive study of all BN-doped fullerene derivatives, $\mathrm{C}_{20-2 n} \mathrm{~B}_{n} \mathrm{~N}_{n}$ $(\forall 1 \leqslant n \leqslant 10)$. We calculated CCSD atomization energy estimates for over 3 million unique species using APDFT2 and identified the most stable system [Fig. 4(c)]. Due to symmetry, all first-order estimates are zero, and only one first-order density perturbation is needed. Figures 4(a) and 4(b) illustrate the quality of the APDFT2 estimates (as well as for MP2, HF, PBE, and PM6) using 100 systems selected at random for all $n$ (for $n=1$ only five compounds exist). Again, the accuracy of APDFT2 decreases with distance in chemical space from the reference molecule, yet remains more accurate than HF or PBE, and even more accurate than MP2 for up to 9 $\mathrm{BN}$ pairs. Due to the small cost of a single density perturbation calculation, the computational speedup is dramatic: Scanning all $\sim 3.1$ million targets requires 8 core days instead of 322 (HF), 54 (PBE), or 977 (MP2) core years. Second, we have optimized the $\pi-\pi$-stacking responsible for the noncovalent bonding to coronene in BN-doped coronene using APDFT3. Figures 4(d)-4(f) illustrate the findings after using a genetic algorithm [56] (see Supplemental Material [49] for more details) to identify systems with maximal attraction and repulsion for every number of BN pairs, among all the $2.8 \times 10^{10}$ possible candidates (not accounting for symmetry). Apparently, the GE achieves strong binding by dialing in a segregation of $\mathrm{B}$ and $\mathrm{N}$ resulting in a large permanent dipole moment, capable of strongly polarizing the other moiety. Validation shows APDFT3 energy estimates to mostly be in the correct ordering, achieving an excellent Spearman rank correlation coefficient of 0.92 (see Supplemental Material [49]), implying

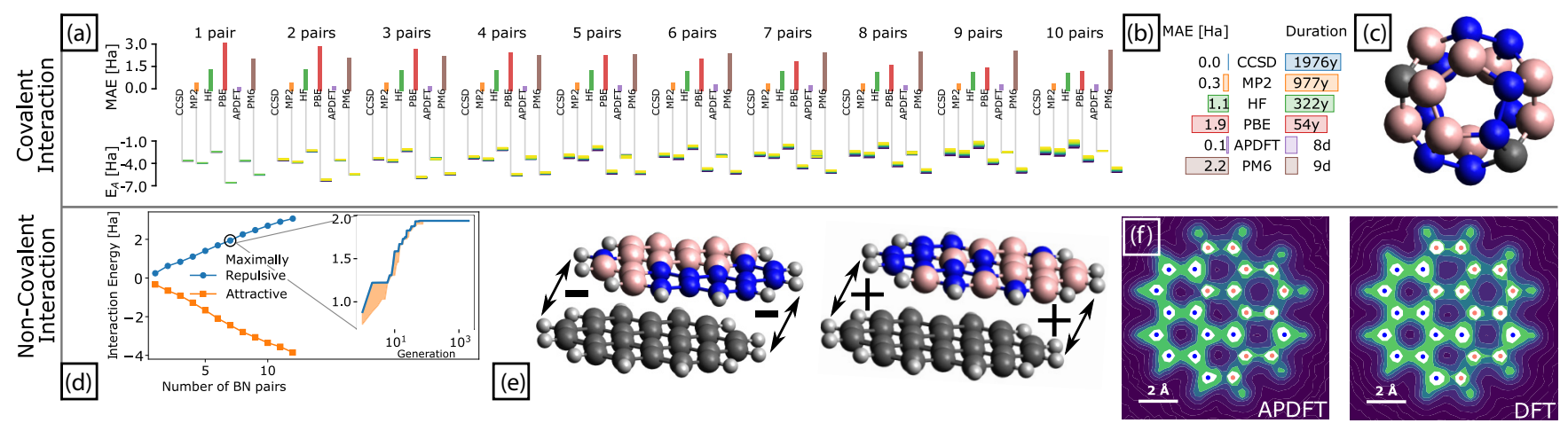

FIG. 4. Energy estimates of covalent [panels (a)-(c), APDFT2] and noncovalent [panels (d)-(f), APDFT3] binding in BN-doped $\mathrm{C}_{20}$ fullerenes and coronene dimers. (a) Mean absolute errors with respect to CCSD/6-31G (MAE), and atomization energy spectra (color corresponding to CCSD rank) for increasing BN content. (b) MAE with respect to CCSD and ballpark CPU time extrapolations (nonoptimal code) for common methods and APDFT2 (see Supplemental Material [49]). (c) Global minimum energy molecule found with APDFT2 (out of $3.1 \times 10^{6}$ systems). (d) Maximal attraction (minimum) and repulsion (maximum) in coronene dimers after genetic evolution (GE) using APDFT3 (with SCAN + VV10/def2-TZVP) [53,54] at every number of BN pairs. For 7 BN pairs, exemplary GE convergence of repulsion maximization is shown (line/shade is best/population), searching $2.8 \times 10^{10}$ possible candidates. (e) Dimers with maximal attraction (-) and repulsion (+) found by GE. (f) Electron density of maximally attractive monomer, obtained from APDFT3 and reference (SCAN + VV10). 
the suitability of APDFT3 to perform even challenging design tasks (noncovalent binding) in chemical space.

\section{CONCLUSION}

To conclude, we introduced an orbital-free alchemical perturbation density functional theory (APDFT). We have shown that the electron density of different target molecules can be constructed such that not only forces but also electrostatic potential density moments are reproduced in the gas phase. We have demonstrated that the converged accuracy of both energy and density is comparable to the level of theory employed for the reference. Consequently, APDFT enables the shift of computational efforts from screening across many molecules at intermediate quality towards a few high-quality calculations. For nondissociative geometries, the accuracy of such predictions converges with perturbation expansion order for all close-by (i.e., $\Delta Z=1$ ) target systems studied.
Similar to forces, the alchemical gradients allow optimization in chemical composition even with a comparably low expansion order, as demonstrated for BN doping of $\mathrm{C}_{20}$ fullerenes and coronene dimers. Similar to using the Hessian used for increasing step lengths in geometry optimizations, alchemical higher orders are required for target systems farther away in chemical space. The numerical results suggest systematic accuracy improvement through inclusion of higher orders, or, conversely, coverage of larger regions of chemical spacefrom one reference perturbation alone.

\section{ACKNOWLEDGMENTS}

We acknowledge support by the Swiss National Science Foundation (Grants No. PP00P2_138932; No. 407540_167186, NFP 75 Big Data; and No. 200021_175747, NCCR MARVEL). This work was supported by a grant from the Swiss National Supercomputing Centre (CSCS) under Project ID s848. Some calculations were performed at sciCORE [57] Scientific Computing Core Facility at University of Basel.
[1] J. Harris, Phys. Rev. B 31, 1770 (1985).

[2] F. W. Averill and G. S. Painter, Phys. Rev. B 41, 10344 (1990).

[3] W. Kohn and L. J. Sham, Phys. Rev. 140, A1133 (1965).

[4] H. M. Polatoglou and M. Methfessel, Phys. Rev. B 37, 10403 (1988).

[5] A. J. Read and R. J. Needs, J. Phys.: Condens. Matter 1, 7565 (1989).

[6] M. W. Finnis, J. Phys.: Condens. Matter 2, 331 (1990).

[7] I. J. Robertson and B. Farid, Phys. Rev. Lett. 66, 3265 (1991).

[8] E. Zaremba, J. Phys.: Condens. Matter 2, 2479 (1990).

[9] D. M. Benoit, D. Sebastiani, and M. Parrinello, Phys. Rev. Lett. 87, 226401 (2001).

[10] W. Zhu and S. B. Trickey, Int. J. Quantum Chem. 100, 245 (2004).

[11] B. Zhou and Y. A. Wang, J. Chem. Phys. 128, 084101 (2008).

[12] L. L. Foldy, Phys. Rev. 83, 397 (1951).

[13] E. B. Wilson, J. Chem. Phys. 36, 2232 (1962).

[14] A. A. Frost, J. Chem. Phys. 37, 1147 (1962).

[15] I. Reif, R. Medina, M. Salazar, and M. García-Sucre, J. Chem. Phys. 81, 1906 (1984).

[16] E. E. Daza and A. Bernal, J. Math. Chem. 38, 247 (2005).

[17] Á. Nagy, Phys. Rev. A 53, 3660 (1996).

[18] Á. Nagy, Chem. Phys. Lett. 460, 343 (2008).

[19] Á. Nagy, J. Chem. Phys. 135, 044106 (2011).

[20] H. Levämäki, Á. Nagy, K. Kokko, and L. Vitos, Phys. Rev. A 92, 062502 (2015).

[21] B. Zhou and Y. A. Wang, J. Chem. Phys. 124, 081107 (2006).

[22] M. Levy, J. Chem. Phys. 68, 5298 (1978).

[23] P. Politzer and R. G. Parr, J. Chem. Phys. 61, 4258 (1974).

[24] J. Linderberg, Phys. Rev. 121, 816 (1961).

[25] F. H. Stillinger, J. Chem. Phys. 45, 3623 (1966).

[26] P. Politzer and K. C. Daiker, Int. J. Quantum Chem. 14, 245 (1978).

[27] E. A. Castro and F. M. Fernández, J. Chem. Phys. 79, 1548 (1983).

[28] M. Levy and Y. Tal, J. Chem. Phys. 72, 3416 (1980).

[29] E. S. Kryachko, Int. J. Quantum Chem. 25, 637 (1984).
[30] O. A. von Lilienfeld, R. D. Lins, and U. Rothlisberger, Phys. Rev. Lett. 95, 153002 (2005).

[31] O. A. von Lilienfeld, J. Chem. Phys. 131, 164102 (2009).

[32] A. Solovyeva and O. A. von Lilienfeld, Phys. Chem. Chem. Phys. 18, 31078 (2016).

[33] D. Alfè, M. J. Gillan, and G. D. Price, Nature (London) 405, 172 (2000).

[34] K. Chang and O. A. von Lilienfeld, CHIMIA Int. J. Chem. 68, 602 (2014).

[35] K. Y. S. Chang, S. Fias, R. Ramakrishnan, and O. A. von Lilienfeld, J. Chem. Phys. 144, 174110 (2016).

[36] A. Beste, R. J. Harrison, and T. Yanai, J. Phys. Chem. 125, 074101 (2006).

[37] M. to Baben, J. O. Achenbach, and O. A. von Lilienfeld, J. Chem. Phys. 144, 104103 (2016).

[38] K. Y. S. Chang and O. A. von Lilienfeld, Phys. Rev. Mater. 2, 073802 (2018).

[39] D. Sheppard, G. Henkelman, and O. A. von Lilienfeld, J. Chem. Phys. 133, 084104 (2010).

[40] K. Saravanan, J. R. Kitchin, O. A. von Lilienfeld, and J. A. Keith, J. Phys. Chem. Lett. 8, 5002 (2017).

[41] F. Weigend, J. Chem. Phys. 141, 134103 (2014).

[42] Y. S. Al-Hamdani, A. Michaelides, and O. A. von Lilienfeld, J. Chem. Phys. 147, 164113 (2017).

[43] M. Lesiuk, R. Balawender, and J. Zachara, J. Chem. Phys. 136, 034104 (2012).

[44] R. Balawender, M. A. Welearegay, M. Lesiuk, F. De Proft, and P. Geerlings, J. Chem. Theory Comput. 9, 5327 (2013).

[45] F. Weigend, C. Schrodt, and R. Ahlrichs, J. Chem. Phys. 121, 10380 (2004)

[46] R. Balawender, M. Lesiuk, F. D. Proft, and P. Geerlings, J. Chem. Theory Comput. 14, 1154 (2018).

[47] S. Fias, F. Heidar-Zadeh, P. Geerlings, and P. W. Ayers, Proc. Natl. Acad. Sci. USA 114, 11633 (2017).

[48] R. P. Feynman, Phys. Rev. 56, 340 (1939).

[49] See Supplemental Material at http://link.aps.org/supplemental/ 10.1103/PhysRevResearch.2.023220 for computational details on the density derivative evaluations and finite difference 
schemes, analysis of numerical stability, proofs of convergence for the hydrogenic atom and free atoms, computational details on the $\mathrm{C}_{20}$ exhaustive search, computational details on the genetic algorithm used for the coronene dimer, BF dissociation curves, and comparison to covalent bonding potentials in Levy's approach.

[50] F. Brockherde, L. Vogt, L. Li, M. E. Tuckerman, K. Burke, and K.-R. Müller, Nat. Commun. 8, 872 (2017).

[51] A. J. Cohen and P. Mori-Sánchez, J. Chem. Phys. 140, 044110 (2014).
[52] M. Levy, Y. Tal, and S. C. Clement, J. Chem. Phys. 77, 3140 (1982).

[53] O. A. Vydrov and T. V. Voorhis, J. Chem. Phys. 133, 244103 (2010).

[54] J. Sun, A. Ruzsinszky, and J. P. Perdew, Phys. Rev. Lett. 115, 036402 (2015).

[55] S. Chakraborty, P. Kayastha, and R. Ramakrishnan, J. Chem. Phys. 150, 114106 (2019).

[56] F.-A. Fortin, F.-M. De Rainville, M.-A. Gardner, M. Parizeau, and C. Gagné, J. Mach. Learn. Res. 13, 2171 (2012).

[57] http://scicore.unibas.ch/. 